\title{
CONTRIBUTION A L'ÉTUDE D'UNE MÉTHODE D'IDENTIFICATION DES LAITS ET FROMAGES AU MOYEN DE L'ÉLECTROPHORÈSE SUR GEL DE POLYACRYLAMIDE
}

\author{
par \\ L. ASSENAT \\ Laboratoire de la Société des Caves et des Etablissements L. Rigal, \\ 12 - Roquefort
}

\section{Avant-propos}

L'objectif du travail présenté ici étant essentiellement la mise au point d'une méthode de contrôle, nous croyons utile de préciser que cette étude, avait, dès l'origine, une portée volontairement limitée. Il ne nous a donc pas été toujours possible de donner une interprétation précise des résultats obtenus et, notamment, en ce qui concerne l'identification de certaines des substances spécifiques.

Le lecteur saura donc juger ce travail en fonction de cet objectif.

\section{Introduction}

Le problème de la garantie s'inscrit dans le cadre de la commercialisation d'un grand nombre de marchandises. Cette considération s'applique dans bien des cas à l'industrie laitière : nécessité de contrôler la nature des produits laitiers de base, souci de livrer sur le marché des produits conformes à l'appellation d'origine.

Notre étude a été envisagée sous l'angle de l'identification des laits de brebis, vache et chèvre ainsi que de leurs produits de transformation, en rapport avec l'industrie fromagère du Roquefort : fromage au lait de brebis. Nous avons abordé ce travail sous l'angle des identifications des protéines du lait et du fromage à partir de l'électrophorèse sur gel de polyacrylamide.

Nous proposons la relation de ce travail d'après le schéma suivant : 
Première partie: chèvre.

Caractérisation et identification des laits de vache, brebis et

Caractérisation et identification des fromages en fonction de leur origine lait.

Deuxième partie:

Adultération de laits entre eux.

Adultération des fromages.

Première partie

\title{
CARACTÉRISATION ET IDENTIFICATION DES LAITS DE VACHE, DE BREBIS ET DE CHÈVRE
CARACTÉRISATION ET IDENTIFICATION
DES FROMAGES EN FONCTION
DE LEUR ORIGINE LAIT

\begin{abstract}
Chapitre premier
Problèmes généraux concernant la caractérisation des différentes espèces de laits et des produits fromagers issus de ces laits,
\end{abstract}

Les produits fromagers, issus de laits d'espèces différentes, ont des caractères étroitement liés à leur origine. Il en résulte des différences importantes dans la valeur marchande des laits et des produits fromagers. D'où la nécessité, pour l'industriel, de s'assurer de l'identité des laits qu'il devra traiter, et, pour l'acheteur, d'avoir une garantie d'origine.

Le souci de disposer de méthodes de contrôle efficaces s'est done manifesté depuis longtemps.

Dans cet ordre idées, nous mentionnerons quelques-unes des études concernant ce problèmes. Citons, pour mémoire, la réaction à l'ammoniaque d'Unikoff, spécialement étudiée par Zimmerman [1], Freudenberg [2] et Alison [3]. Cette réaction n'a d'ailleurs qu'une faible valeur de spécificité.

Carrie [4], a établi que les indices de Polenske (acides gras volatils) du lait de vache et de brebis, étaient respectivement de 3,35 et 6,25 . 
Nombre de chercheurs se sont intéressés aux méthodes sérologiques. Bordet en 1899 [5], Fisch en 1939 [6], produisaient des sérums anti-laits eapables de précipiter la caséine du lait, mais ils obtinrent des réactions qui n'étaient pas strictement spécifiques. Fordenoff et Koschueharoff en 1939 [7], Legros en 1949 [8], étudièrent, dans le même sens, des techniques de détection et dosage rapide de laits animaux frauduleusement ajoutés au lait de femme. En Norvège, Solberg et Hadland en 1953 [9], utilisaient des sérums anti-laits pour détecter les fraudes de lait de chèvre dans le lait de vache. Plus récemment, Dutheil en 1959 [10], au Laboratoire de Roquefort, proposait une méthode basée sur ce principe, pour la détection des fraudes de lait de chèvre ou de vache dans le lait de brebis, et Nair et Iya aux Indes [11], eelle du lait de vache dans le lait de buflesse. Signalons également la méthode sérologique d'identification de Pinto, 1966 ([11bis].

On sait depuis longtemps - Alais [12] — que le diagramme électrophorétique des laits (caséines et protéines solubles), est caractéristique de chaque espèce animale. Les caséines étaient, jusqu'à une époque relativement récente, assez difficiles à séparer; c'est done surtout sur les protéines du sérum - $\beta$-lactoglobuline, $\alpha$-lactalbuminequ'ont porté les investigations. Biserte et coll. en 1953 [13], ont étudié par électrophorèse les séroprotéines du lait de femme et de vache. Ambrosino et coll. [14], publièrent en 1961 une étude des protéines du sérum des laits de brebis, vache et buflesse, étude effectuée conjointement par chromatographie et par électrophorèse sur gel d'amidon. Hilpert et Enkelmann en 1963 [15], publièrent à partir de l'électrophorèse sur papier des séroprotéines, des conclusions sur les possibilités de caractérisation des laits de chèvre, vache, lapine et cobaye.

C'est dans une perspective du même ordre que nous avons envisagé de tirer parti des avantages du procédé d'électrophorèse sur gel de polyacrylamide.

Des préoccupations de même nature s'appliquent au fromage. Les méthodes pratiques de différenciation des fromages sont peu nombreuses et imprécises, surtout lorsqu'il s'agit d'identifier des mélanges. L'une de ces méthodes est basée sur le dosage des acides volatils solubles et insolubles : indices de Reichert-Meissl-Wollny et indice de Polenske. Les réactions biologiques ont été exploitées sans grand succès. Nous avons pensé que la méthode électrophorétique sur gel d'acrylamide pourrait être un moyen de parvenir à une solution.

Les matières azotées du fromage forment, avec les lipides, l'essentiel de la matière sèche du fromage. La caséine représente 90 à 95 p. 100 des protéines totales des produits frais mais une partie se dégrade au cours de l'affinage, fournissant ainsi des produits d'hydrolyse : protéose, peptides, acides aminés. 
Lindqwist et Storgards [16], ont étudié, par électrophorèse, les modifications de la caséine de vache au cours de la maturation du fromage et conclurent à " différents stades d'un processus unique de la décomposition de la caséine ". Nous en avons déduit que, si ce processus est unique pour une caséine d'origine définie - fromages à base de lait de vache - les produits dérivés du lait d'autres espèces pourraient avoir également, à l'affinage, un comportement spécifique. Ceci résulterait logiquement de la nature différente des protéines des laits d'origine (vache, brebis, chèvre, etc.).

\section{Chapitre II}

\section{Electrophorèse sur gel de polyacrylamide. Matériel et méthodes}

La méthode de travail que nous avons adoptée se rapporte pour l'essentiel, au procédé d'étude des protéines du lait décrit par Aschaffenburg [17]. Il est utilisé depuis quelque temps en France par les chercheurs des Laboratoires du C.N.R.Z. : Garnier J., Ribadeau-Dumas B., et collaborateurs. Il donne d'excellentes séparations électrophorétiques et son application est simple, pratique et peu onéreuse.

\section{Appareillage.}

Le cadre dans lequel est coulé le gel est constitué par une plaque de plastique $(17,5 \times 9 \times 0,3 \mathrm{~cm})$ unie à une plaque de base $(20 \times 11,5 \times 0,3 \mathrm{~cm})$, entourée d'un cadre détachable de $0,6 \mathrm{~cm}$ d'épaisseur. L'ensemble constitue un petit bac démontable de faible épaisseur dont les dimensions internes sont: 17,5 $\times 9 \times 0,3 \mathrm{~cm}$ (voir schéma figure 1).

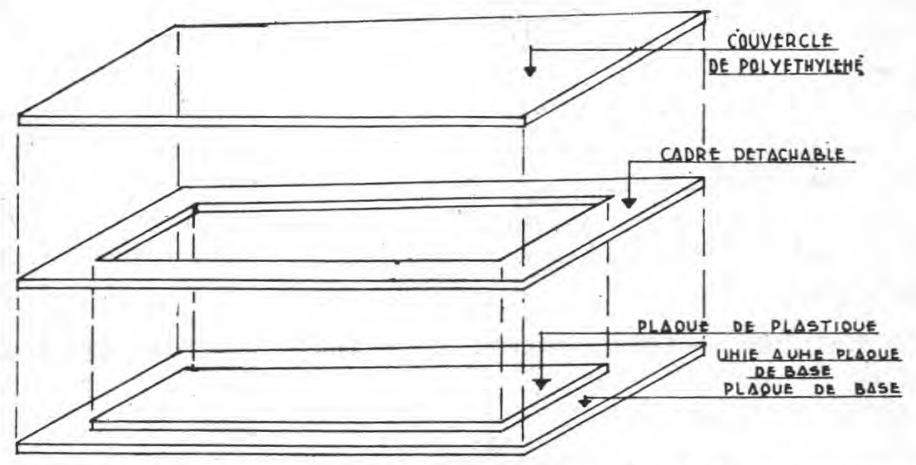

Fig. 1. - Cadre support pour électrophorèse sur gel de polyacrylamide (selon Aschaffenburg) 
Les bacs à tampon sont d'un type courant, avec électrodes incorporées connectées à un générateur redresseur de courant 500 V-100 mA.

Pour'la révélation, nous utilisons un jeu de bacs rectangulaires $(30 \times 20 \times 10 \mathrm{~cm})$, et pour la lecture un photomètre enregistreur.

\section{Réactifs}

1) Solution stock de tampon véronal $p \mathrm{H} 8,6$ :

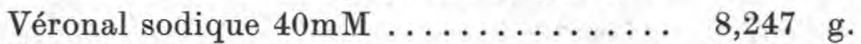

Véronal $8 \mathrm{mM} \ldots \ldots \ldots \ldots \ldots \ldots \ldots \ldots \ldots \ldots, 1,473 \quad 9$.

Eau distillée q.s.p. ............. $1000 \mathrm{ml}$.

Dissoudre d'abord le véronal par agitation dans une quantité suffisante d'eau distillée et ajouter ensuite le véronal sodique.

2) Solution stock d'acrylamide :

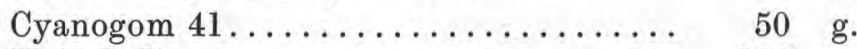

Urée R.P................. 300 g.

Solution DMAPN à 10 p. $100 \ldots \ldots \ldots \ldots .20 \mathrm{ml}$.

Tampon véronal stock $(p \mathrm{H} 8,6)$

dilué au 1/10 q.s.p. ........... $1000 \mathrm{ml}$.

Cette préparation doit être conservée au froid. Limiter le stockage à huit-dix jours.

3) Solution de persulfate d'ammonium à 10 p. 100 :

Renouveler cette solution tous les huit jours.

\section{Technique}

\section{Préparation des gels.}

Chacun des cadres a été prévu pour une contenance de $60 \mathrm{ml}$. Pour chaque fraction de $60 \mathrm{ml}$ de solution stock d'acrylamide, ajouter $1 \mathrm{ml}$ de la solution de persulfate d'ammonium (catalyseur de gélification). Verser le liquide dans le support. La réaction de polymérisation ne s'effectuant qu'en l'absence d'oxygène, recouvrir le cadre avec une plaque de polyéthylène de $3 \mathrm{~mm}$ d'épaisseur et éviter d'emprisonner les bulles d'air. Laisser gélifier au moins trois heures. Le gel urée est spécialement utilisé pour la séparation des caséines. L'électrophorèse des protéines solubles est pratiquée sur gel identique au précédent mais dépourvu d'urée.

\section{Electrophorèse.}

Le couvercle en polyéthylène est retiré du cadre. Une fente distante de $6 \mathrm{~cm}$ de l'extrémité cathodique de la plaque est pratiquée avec une lame de rasoir. A l'aide d'une fine spatule, écarter les lèvres de la fente et insérer dans le gel les languettes de papier Whatman 3M 
$(1,5 \times 0,30 \mathrm{~cm})$ imbibées du produit à tester. Pour chaque gel, quatre languettes peuvent être ainsi disposées, chacune étant séparée de l'autre par un espacement de $2 \mathrm{~mm}$ environ.

La plaque est mise sur le dispositif de telle sorte que les deux extrémités soient supportées par le bord interne de chacune des cuves à tampon et que la migration s'effectue dans le sens cathodeanode. Avec le matériel dont nous disposons, on peut grouper jusqu'à 4 plaques à la fois. Les connexions avec le liquide des bacs électrodes sont faites avec une double couche de papier Whatman 3M. Les ponts sont humectés avec la solution véronal stock, laquelle emplit chacun des bacs. Les cadres sont eux-mêmes recouverts de la plaque de polyéthylène épaisse (voir schéma fig. 2).

Appliquer un courant constant de $12 \mathrm{~mA}$ par gel et un voltage initial de 100 volts, ceci de préférence à la température d'une chambre froide ou d'une armoire frigorifique (4 à $\left.8^{\circ} \mathrm{C}\right)$. Temps de migration : environ 8 heures.
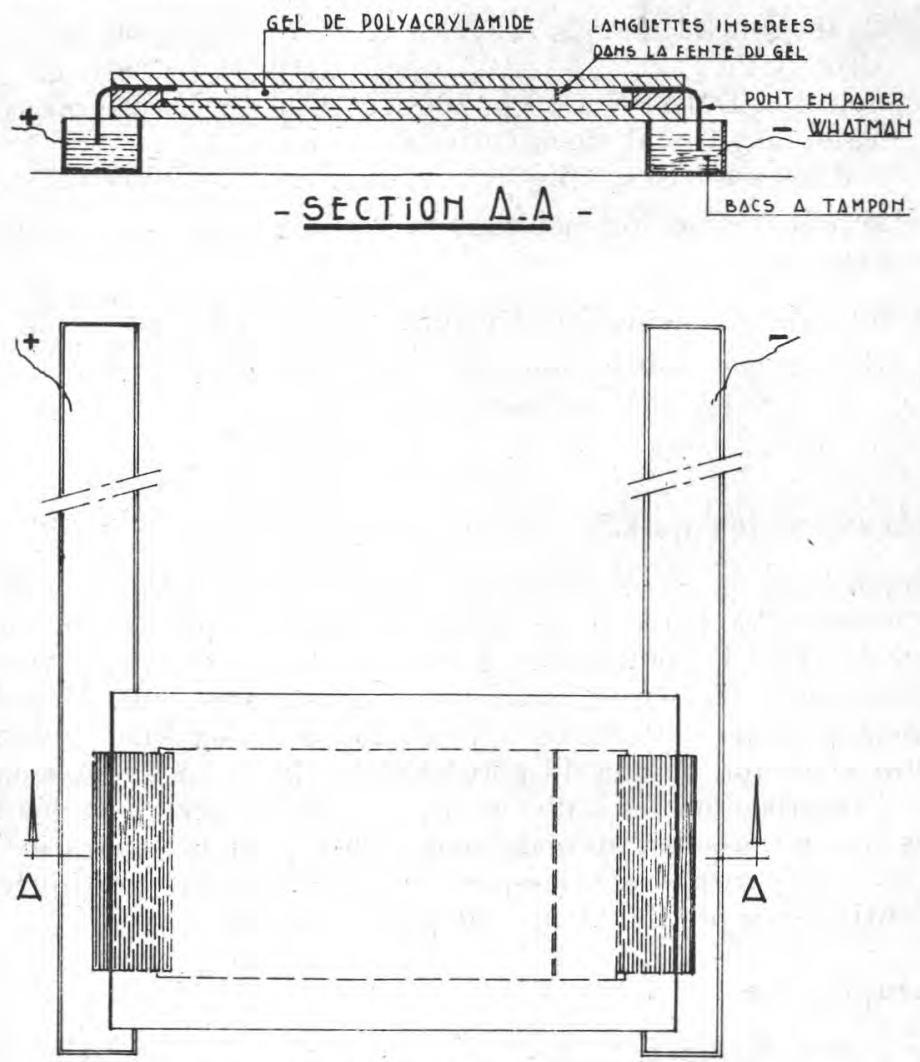

- VUE EH PLAY -

Fig. 2. - Dispositif d'électrophorèse sur gel de polyacrylamide. 
Il peut arriver que les vitesses de migration ne soient pas identiques d'une expérience à l'autre (influence des variations de température, d'intensité de courant, etc.). Un bon moyen, pour corriger cet inconvénient, consiste à appliquer, sur l'un quelconque des rectangles humectés, une légère touche d'éosine poudre. Le traceur coloré migre de façon visible à l'avant du spectre des oaséines et il suffit d'arrêter l'électrophorèse, lorsque la bande colorée atteint l'autre extrémité de la plaque, pour avoir des migrations toujours comparables.

Pour les études des protéines solubles ( $\beta$-lactoglobulines et $\alpha$ lactalbumines), on effectue les électrophorèses sur gel sans urée, à température ordinaire, avec $12 \mathrm{~mA}$ par gel pendant 4 heures.

\section{Révélation.}

On retire le couvercle et le cadre. Le gel est détaché de son support et placé, pour 3 minutes environ, dans le bac contenant la solution colorante de formule suivante :

\section{Solution colorante}

Amido-Schwartz .................

Acide acétique...................

$1 \mathrm{~g}$.

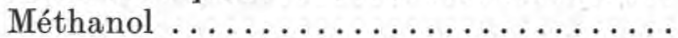

$100 \mathrm{ml}$.

Eau distillée q.s.p. .............. $1000 \mathrm{ml}$.

Cette solution peut être réutilisée une cinquantaine de fois. Le gel est ensuite placé dans une solution d'acide acétique à 10 p. 100. Renouveler jusqu'à décoloration totale.

Si le gel doit être conservé, faire un lavage final pendant 1 à 2 heures avec la solution durcissante de formule suivante:
Méthanol ................... $300 \mathrm{ml}$.
Eau distillée q.s.p................, $600 \mathrm{ml}$.
Acide acétique ................ $100 \mathrm{ml}$.

\section{Dessiccation et conservation du gel.}

Dans la plupart des cas il est utile de conserver les résultats des électrophorèses et de faire leur relevé graphique.

Les auteurs conseillent de les étaler sur papier Whatman 3M et de les laisser sécher à l'air, mais il est difficile de les utiliser pour une révélation graphique ultérieure et les gels ainsi traités ont une tendance marquée à se rouler et à devenir cassants, même conservés sous presse.

Nous avons adopté une technique différente : sur un rectangle rigide et de faible épaisseur, en métal ou plastique, de $18 \times 12 \mathrm{~cm}$, disposer une feuille de cellophane humide, de dimensions telles 
qu'elle déborde de $5 \mathrm{~cm}$ environ sur tout le périmètre; rabattre les marges et les plier par dessous le support - l'humidité de la cellophane les maintient en place - déposer le gel à son tour, mettre en place une nouvelle feuille de cellophane, dans les mêmes conditions que la première fois, de façon à emprisonner le gel entre les deux feuilles. Chasser les inclusions de gaz qui ont pu se former au cours de l'opération. Au besoin, faire une ou deux piqûres d'épingle pour chasser l'air emprisonné entre les deux feuilles. Dessécher en atmosphère ambiante ou mieux à proximité d'un petit ventilateur. On obtient un gel desséché, glacé, transparent et utilisable dans les meilleures conditions.

\section{Interprétation des résultats.}

D’une façon générale, les substances séparées sont identifiées par rapport à un repère de base. Comme les distances de migration dépendent $d u$ temps et d'autres facteurs, qui ne sont pas toujours contrôlables, on calcule la migration d'une substance ( $R x$ ) par rapport à une substance témoin à laquelle on a donné conventionnellement la valeur I. Donc pour une substance quelconque, on calculera le Rx d'après la formule :

$\mathrm{Rx}$ (ou Indice de mobilité) $=\frac{\text { Distance parcourue par la substance }}{\text { Distance parcourue par le témoin }}$

Dans le cas particulier des caséines du lait, nous avons adopté le système de calcul des indices de mobilité selon Wake et Baldwin [18]. La position de la substance témoin à laquelle les auteurs donnent la valeur I, est celle d'un composant mineur situé sur le spectre des caséines du lait de vache sitôt après la bande des caséines.

Les relevés graphiques au photomètre enregistreur permettent l'évaluation des quantités relatives de substances séparées.

\section{Préparation des échantillons.}

Lait entier.

L'électrophorèse de lait entier sur gel de polyacrylamide est couramment utilisée. Elle permet la séparation de tous les composants protéiniques à poids moléculaire élevé, mais convient particulièrement pour l'étude des caséines. Le lait est utilisé dilué au $1 / 4$ avec une solution d'urée 5M.

\section{Caséines du lait.}

Le lait préalablement écrémé est dilué au 1/5 avec de l'eau distillée, chauffé à $35^{\circ} \mathrm{C}$, et additionné d'acide acétique $\mathrm{N}$ jusqu'à $p H$ 4,6. La caséine précipitée est recueillie par centrifugation et lavée plusieurs fois. 
La caséine recueillie est reprise avec une solution d'urée $7 \mathrm{M}$, et amenée à solubilisation complète par agitation et addition lente de soude $\mathrm{N}$ jusqu'à $p \mathrm{H} 7$.

\section{Protéines solubles.}

Les protéines solubles du lait se retrouvent dans le filtrat de précipitation acétique des caséines : méthode Rowland-Aschaffenburg [19-20].

\section{Caséines $x$.}

Dans la pratique habituelle de l'électrophorèse, des caséines en milieu urée, les variants $x$ n'apparaissent pas clairement sur le spectre. La réduction des ponts disulfure, réalisée par l'utilisation $d u \quad \beta$ mercapto-éthanol, permet d'obtenir une bonne séparation électrophorétique de ces composants. Cependant, la caséine $x$ ne représente que 15 à 20 p. 100 de la caséine totale et, de ce fait, n'apparaît à l'électrophorèse que d'une façon relativement faible lors d'examen de caséine entière.

Pour l'étude comparative des caséines $x$ des laits de différentes espèces, nous utilisons la méthode simplifiée d'enrichissement en caséine $x$ d'après Zittle et Custer [21].

- $100 \mathrm{ml}$ de lait, écrémé, dilué au $1 / 2$, réchauffé à $30^{\circ} \mathrm{C}$ et précipité à $p \mathrm{H} 4,6$ avec $\mathrm{HCl} \mathrm{N}$.

- Centrifuger. Recueillir le culot et le dissoudre avec $25 \mathrm{ml}$ d'urée $7 \mathrm{M}$.

- Acidifier avec $5 \mathrm{ml}$ d'acide sulfurique $7 \mathrm{M}$ (1 partie concentrée plus 4 parties d'eau). Ajouter $50 \mathrm{ml}$ d'eau distillée $(p H \mathbf{H} 1,3-1,5)$. Laisser au repos 2 heures. Centrifuger.

- Recueillir le surnageant. Ajouter 13,2 g de sulfate d'ammonium pour $100 \mathrm{ml}$. Centrifuger.

- Recueillir le précipité. Dissoudre dans $20 \mathrm{ml}$ d'eau distillée et $\mathrm{NaOH} \mathrm{N}$ jusqu'à $p \mathrm{H}: 7-7,5$.

- Ajouter à la solution 2 fois son volume d'éthanol et $1 \mathrm{ml}$ de solution saturée d'acétate d'ammonium dans l'éthanol. Attendre $1 / 2$ heure. Centrifuger.

- Reprendre le culot avec $2 \mathrm{ml}$ d'une solution à 1 p. 100 de mercapto-éthanol.

\section{Extraction des protéines précipitables du fromage.}

- Peser 10 grammes de fromage dans un erlenmeyer;

- Ajouter $30 \mathrm{ml}$ d'eau froide ;

- Mélanger à l'aide d'un appareil mixeur à bras dispersant (genre Ultra-Turrax). Le barattage intense permet la séparation sous forme d'un amas de beurre, de la plus grande partie de la matière grasse ; 
- Séparer le beurre de la suspension de caséines ;

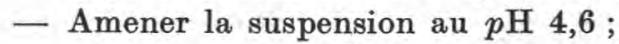

- Centrifuger à 3000 tours/mn pendant $3 \mathrm{mn}$. Rejeter le surnageant ;

- Reprendre le culot avec $5 \mathrm{ml}$ d'urée 7M (420 g/litre);

- Activer la dissolution des caséines à l'aide d'un agitateur. Amener à $p \mathrm{H} 7$;

- Toujours dans le godet de centrifugeuse ajouter $20 \mathrm{ml}$ de tétrachlorure de carbone. Agiter fortement;

- Centrifuger. Elimination de la matière grasse résiduelle.

- La solution surnageante est prête à l'emploi. Au besoin, conserver deux ou trois jours au froid.

Cette formule d'extraction est valable pour toutes les catégories de fromages. Il convient seulement d'ajuster la prise d'essai à la quantité de protéines précipitables présentes : 10 grammes pour fromages affinés (40 à 60 p. 100 de matières azotées solubles), 5 grammes pour fromages peu affinés (20 à 40 p. 100 de matières azotées solubles), 2 grammes pour fromages frais ou demi-frais.

Extraction des protéines précipitables sur dressings.

Les formules de dressings sont très variées. Il convient d'adapter la technique d'extraction à chaque cas particulier. Nous faisons mention, ici, de la technique que nous employons le plus couramment. distillée ;

- Peser $30 \mathrm{~g}$ de dressing dans un bécher. Ajouter $30 \mathrm{ml}$ d'eau

- Amener à $p \mathrm{H} 4,6$, avec acide acétique $\mathrm{N}$;

- Centrifuger $5 \mathrm{mn}$ à 3000 tours $/ \mathrm{mn}$;

- Reprendre le culot avec de l'eau distillée. Laver deux ou trois fois ;

- Reprendre le précipité avec $5 \mathrm{ml}$ d'Urée 7M. Ajuster à $p H$ 7. Ajouter $20 \mathrm{ml}$ de tétrachlorure de carbone. Agiter et centrifuger ;

- Recueillir la fraction surnageante pour électrophorèse.

\section{Chapitre $I I I$}

\section{Etude électrophorétique des laits de vache brebis et chèvre}

\section{Caséines $\alpha_{\mathrm{s}}$ et $\beta, \beta$-lactoglobulines, $\alpha$-lactalbumines.}

Les différentes études ont permis d'établir que les variants caséines ainsi que les $\beta$-lactoglobulines et $\alpha$-lactalbumines avaient des positions invariables sur le spectre électrophorétique. Au cours 
de ce travail, nous avons, le plus possible, utilisé des laits de grand mélange.

L'électrophorèse de laits entiers sur milieu urée, fournit un spectre dans lequel sont présentes toutes les protéines : caséines et protéines solubles. La figure 3 reproduit les électrophorèses comparées de laits de vache, brebis et chèvre. Le spectre de chacune de ces catégories de lait est de toute évidence différent de celui des autres.
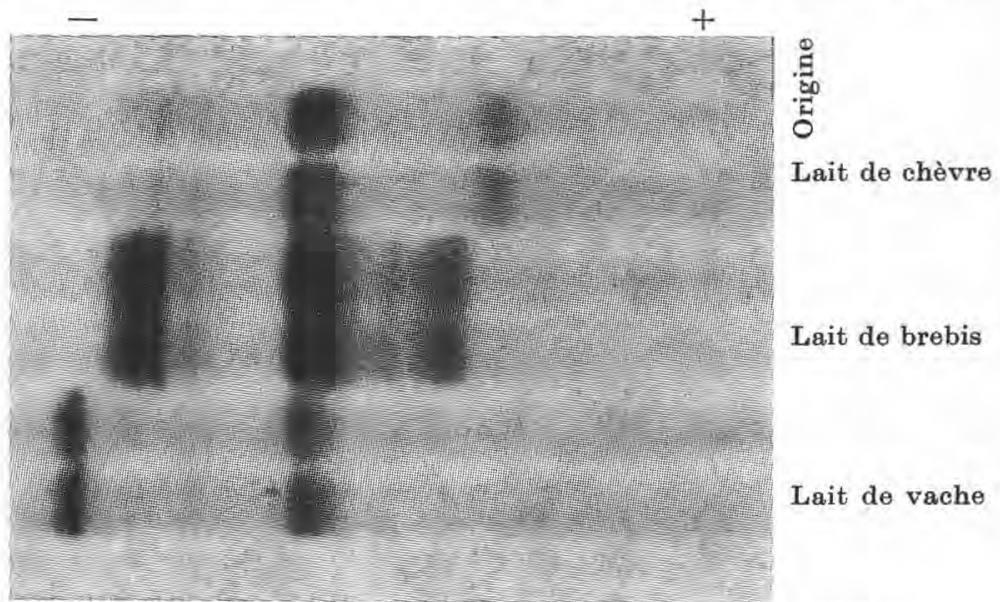

Lait de brebis

Lait de vache

Fig. 3 (1). - Electrophorèses comparées de laits entiers.

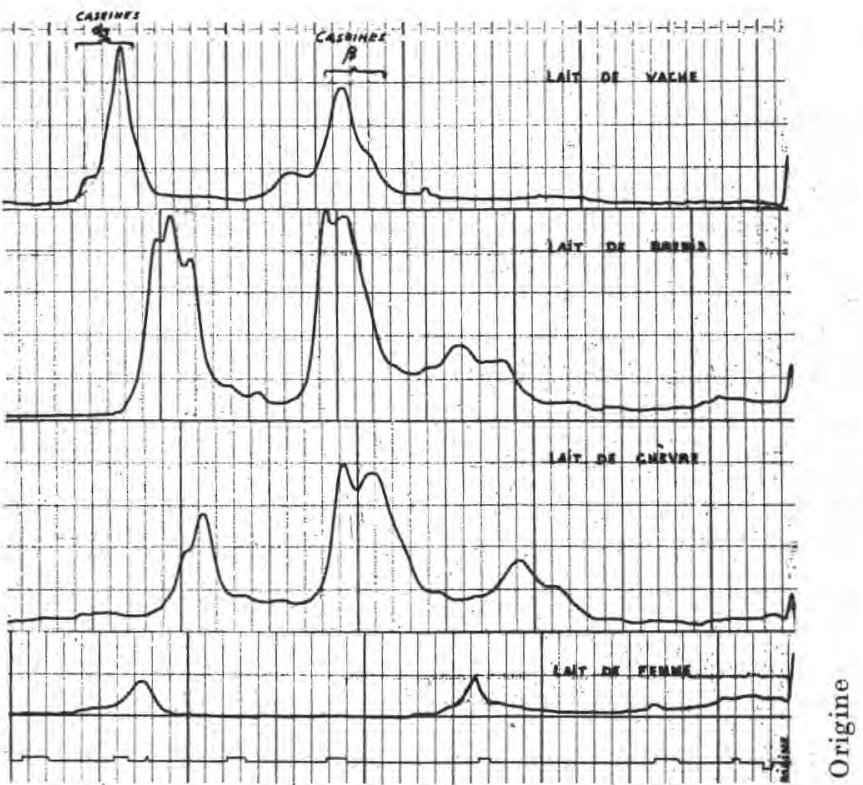

Fig. 3 (2). - Diagrammes d'électrophorèses de laits entiers. 
Nous avons relevé les indices de mobilité des bandes de chacun des spectres par rapport à la substance d'indice de mobilité I du lait de vache, défini par Wake et Baldwin [18]. Pour simplifier les interprétations, nous avons d'abord relevé les $\mathrm{Rx}$ des caséines $\alpha_{\mathbf{s}}$ et $\beta$.

\section{Indices de mobilité des caséines}

\begin{tabular}{|c|c|c|c|c|c|}
\hline \multirow{3}{*}{ Caséine } & \multirow{2}{*}{\multicolumn{2}{|c|}{$\alpha_{\mathrm{s}} \quad \mathrm{A}$}} & vache & brebis & \multirow[t]{2}{*}{ chèvre } \\
\hline & & & & 1,01 & \\
\hline & & B & 1,09 & 0,97 & 0,97 \\
\hline & & C & 1,06 & 0,95 & 0,95 \\
\hline Caséine & $\beta$ & A & 0,70 & 0,73 & 0,73 \\
\hline & & B & 0,67 & 0,68 & 0,68 \\
\hline
\end{tabular}

Les substances que nous qualifions de caséines ont été identifiées comme telles d'après leur caractère de précipitabilité à $p \mathrm{H} \mathrm{4,6}$ (figure 4 : caséines).

Nous avons ensuite fait l'identification des caséines individuelles $\alpha_{\mathrm{s}}$ et $\beta$ par la méthode de précipitation fractionnée au calcium.

Outre les principales formes de caséines, l'électrophorèse de lait entier met en évidence d'autres substances : $\beta$-lactoglubulines $\alpha$-lactalbumines. Nous ne les avons pas identifiées individuellement mais avons réalisé les électrophorèses séparées de ces substances (fig. 4 : protéines solubles) sur lactosérum de chacun des laits considérés. Nous avons noté le $\mathrm{Rx}$ des plus importantes : 0,51 et 0,45 , pour le lait de brebis, 0,36 pour lait de chèvre.

\section{II. - Discussion.}

La première observation générale que nous avons formulée concerne le comportement électrophorétique spécifique des laits de vache, brebis et chèvre.

Plusieurs centaines d'échantillons de chacun des laits ont été examinés; leurs spectres d'électrophorèse sont invariablement identiques pour chaque espèce.

L'examen détaillé des électrophorèses de caséines nous apprend que :

1) Le Rx des caséines $\alpha_{\mathrm{s}}$ de vache est nettement plus important que celui des caséines $\alpha_{\mathbb{S}}$ de brebis et de chèvre : $1,09,1,06$ contre $1,01,0,95$.

2) Le $R x d u$ variant $\alpha_{S}$ le plus lent (C) du lait de vache est plus grand que le $\mathrm{Rx}$ le plus important des variants caséines $\alpha_{\mathrm{g}}$ (A) des laits de brebis et de chèvre : 1,06 contre 1,01 et 0,97.

3) Les $\mathrm{Rx}$ des caséines $\alpha_{\mathrm{s}}$ et $\beta$ de brebis et de chèvre sont sensiblement identiques. 


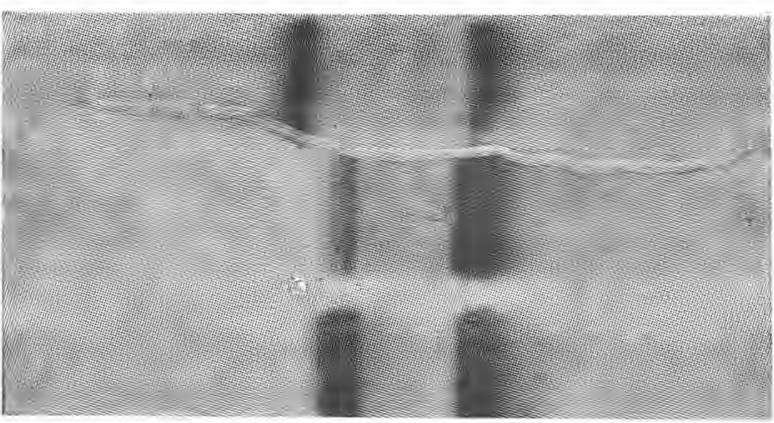

Caséines

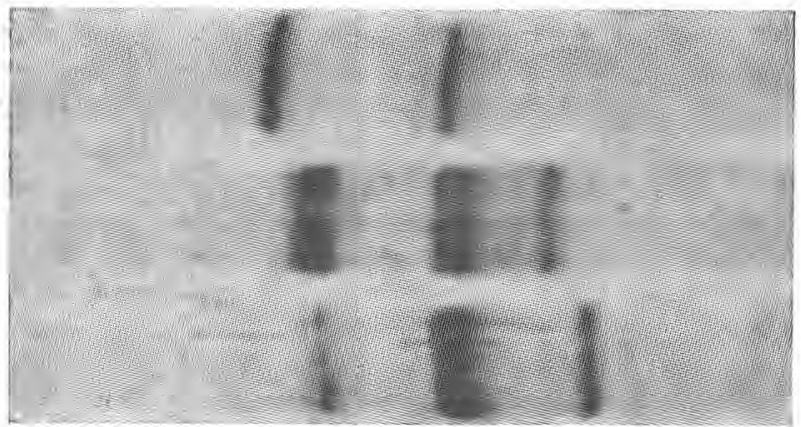

Laits entiers

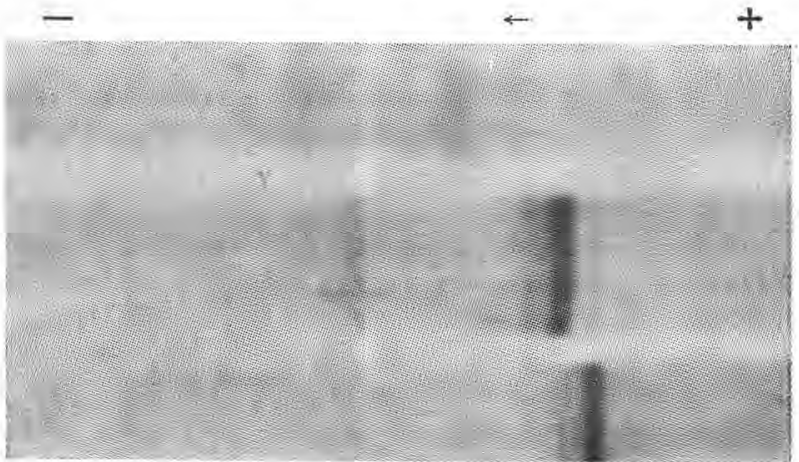

Protéines solubles
Lait de vache

Lait de ehèvre

Lait de brebis

Lait de vache

Lait de brebis

Lait de chèvre

Lait de vache

Lait de brebis

Lait de chèvre

Fig. 4. - Electrophorèses séparées de caséines et protéines solubles 
Les protéines solubles de ces laits ont également des caractéristiques liées à leur origine animale.

1) Les $\beta$-lactoglobulines du lait de vache migrent à l'avant de $\alpha_{\mathrm{s}}$-caséines avec un $\mathrm{Rx}$ de 0,76 .

2) Les principales protéines solubles des laits de brebis et de chèvre ont des $R_{X}$ de $0,51-0,44$ pour les premiers, $0,44-0,37$ pour les seconds. Elles ont par ailleurs un profil électrophorétique très marqué.

\section{III. - Caséines $\varkappa$.}

Nous avons appliqué aux laits de brebis et de chèvre le procédé d'extraction de la caséine $x$ ou plutôt d'enrichissement de cette caséine, d'après Zittle et Custer [21]. Ainsi que nous le verrons ultérieurement, cette opération permet de mettre en évidence les caséine $x$ de vache, de brebis et de chèvre. Comme l'ont signalé les auteurs ci-dessus [22], sur leur étude de la caséine chèvre, cette catégorie de caséine est mise en évidence à partir de la coagulation du lait par la présure. Sur lait de chèvre cru, la caséine $x$ a une mobilité électrophorétique très voisine de celle des caséines $\beta$. Cette caséine est done difficilement identifiable dans ce cas. Les résultats obtenus à partir de préparations de lait traité à la présure font apparaître une bande de mobilité inférieure; cette bande correspond à la para-caséine $x$.

Nous présentons deux groupes d'électrophorèses d'extraits de caséine $x$ de lait de vache, brebis, chèvre, le premier (fig. 5) sur lait normal, le second (fig. 6) sur le même lait soumis à l'action de la présure.

Sur le spectre du lait de vache normal, nous retrouvons, outre les résidus des caséines $\alpha_{\mathrm{s}}$ et $\beta$ que nous négligerons $\left(\mathrm{Rx}_{\mathrm{x}} 0,60\right)$ un certain nombre de bandes de $\mathrm{Rx}_{\mathrm{x}} 0,55$ à 0,35 et qui sont bien connues comme étant des caséines $x$, Les caséines $x$, après action de la présure sur le même lait et donc après élimination du caséinomacropeptide, se retrouvent (fig. 6) sur la position cathodique de Rx-0,60. Nous noterons déjà, sur lait avant coagulation, la présence d'une petite quantité de cette caséine, à la position susmentionnée (décomposition spontanée de la liaison sensible à la présure ou à l'action enzymatique des microorganismes).

Pour ce qui est de la caséine $x$ du lait de brebis, sa position électrophorétique diffère de celle du lait de vache. Elle semblerait se situer à un $\mathrm{Rx}$ plus élevé et très proche de celui des caséines $\beta$. Après action de la présure, cette même caséine apparaît dans la zone cathodique avec le $\mathrm{Rx}-0,30$. 
Le même traitement, appliqué au lait de chèvre, permet de mettre en évidence la para-caséine $x$ dont le $\mathrm{Rx}$ spécifique a comme valeur $-0,08$.

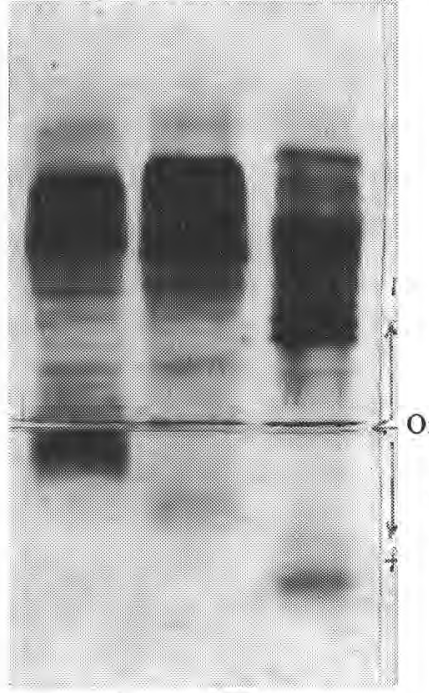

Fig. 5

Laits frais

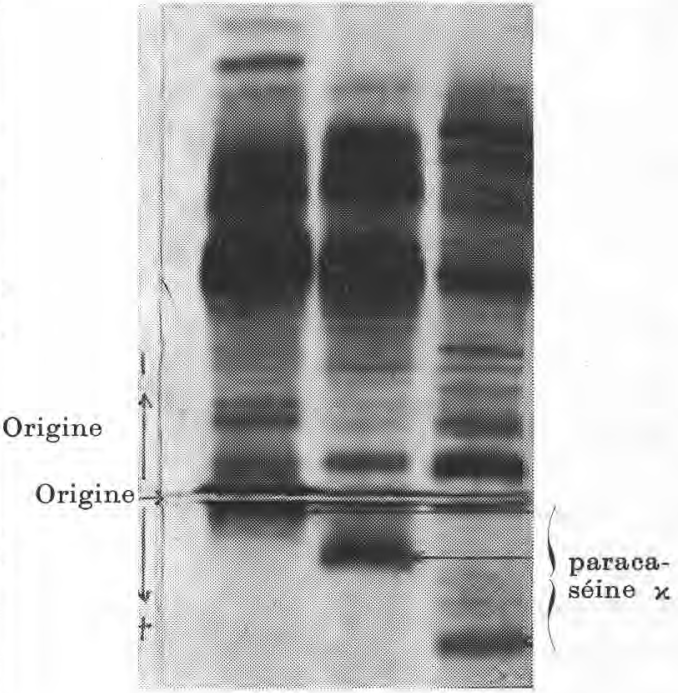

Fig. 6

Laits caillés présure

(Pour chaque groupe d'électrophorèses et de gauche à droite : lait de chèvre, lait de brebis, lait de vache)

\section{IV. - Conclusion. Identification des laits.}

De l'ensemble de ces observations, nous retiendrons que l'identification des laits, par la méthode d'électrophorèse sur gel est suffisamment sûre pour l'utiliser pratiquement dans bon nombre de cas.

1) Le lait de vache sera caractérisé d'après:

- La position des caséines $\alpha_{\mathrm{s}}$ spécifique de ce lait;

- La position des caséines $x$ extraites du lait normal ;

- La position des caséines $x$ extraites du lait ayant subi l'action de la présure. Cette position est également spécifique du lait de vache ;

- La position des $\beta$-lactoglobulines et des $\alpha$-lactalbumines.

2) Le lait de brebis est identifié à partir des éléments suivants:

- La position des caséines $\alpha_{\mathrm{s}}$. Cette position commune à celle des caséines $\alpha_{\mathrm{s}}$ chèvre, est différente de celle des caséines $\alpha_{\mathrm{s}}$ de vache. 
- La position des caséines $x$ extraites du lait ayant subi l'action de la présure. Cette position est spécifique du lait de brebis.

- La position des $\beta$-lactoglobulines du lacto-sérum.

3) Le lait de chèvre est identifiable à partir des éléments suivants :

- La position des $\alpha_{\mathrm{S}}$ caséines, commune au lait de chèvre et de brebis, mais différente du lait de vache. Nous remarquons, toutefois, que les caséines $\alpha_{\mathrm{s}}$ de chèvre et de brebis ne sont pas parfaitement identiques et que la quantité de caséine $\alpha_{\mathrm{s}}$ chèvre est toujours relativement faible.

- La position de la caséine $x$ ayant comme valeur de $\mathrm{Rx}-0,08$ est une substance spécifique du lait de chèvre.

- La position des $\beta$-lactaglobulines, très différenciée vis-à-vis du lait de vache, est cependant assez voisine de celles du lait de brebis.

\section{Remarques :}

Les échantillons de lait susceptibles d'être soumis à ce genre d'examen peuvent être conservés sans inconvénients avec le bichromate de potasse, ou le bichlorure de mercure.

Par ailleurs, un échantillon ayant subi une coagulation lactique et à condition qu'il ne soit pas trop détérioré, peut être examiné dans les mêmes conditions.

S'il a subi la coagulation présure, on l'identifiera d'après les critères définis pour le fromage et dont nous parlerons ultérieurement.

\section{Chapitre IV}

\section{Etude électrophorétique des fromages}

Cette étude avait, comme objectif, l'identification du fromage d'après le lait d'origine. Nous avons plus spécialement examiné deux catégories de fromages bien définies :

- Le Roquefort, fabriqué exclusivement avec du lait de brebis;

- Le Bleu de vache.

Nous avons ainsi abordé l'étude des protéines du fromage ayant les caractéristiques de la caséine. Parmi les composants azotés du fromage nous avons donc retenu ceux d'entre eux qui sont classés dans la catégorie de la matière azotée non soluble et que l'on considère communément comme étant de la caséine : substances précipitables à $p H 4,6$. Il s'agit, en fait, pour une part, de caséines natives et pour l'autre part, de caséines ayant déjà subi un début de modification protéolytique. 


\section{I. - Fromages de Roquefort et Bleu de vache affinés.}

Le cliché (fig. 7) reproduit les électrophorèses comparées de Roquefort et Bleu des Causses, dont le taux d'affinage se situe respectivement à 51,3 et 49,8 p. 100 de matière azotée soluble. Le relevé photométrique, (fig. 8) permet de situer la position et l'importance relative de chacune des protéines séparées.

Nous y notons des spectres très différents. Nous remarquons également la présence d'un nombre de substances azotées plus important que dans le lait.

Comme pour les protéines des laits, nous avons adopté ici le système d'identification des indices de mobilité, selon Wake et Baldwin.

\section{Caséines vraies.}

Les caséines originelles n'existent plus sous cette forme que dans de faibles proportions. Elles ont conservé, sur le spectre, leur position d'origine.

\section{Caséines modifiées.}

Les substances dérivées représentent une part importante des protéines précipitables. Elles sont de nature variée. Certaines d'entre elles nous intéressent en raison de la spécificité qui les relie à l'espèce dont elles proviennent. Nous avons retenu pour chacune des catégories de fromage celles des principales substances spécifiques dont le Rx est le plus marquant.

\section{Fromage Bleu de vache}

1) Protéine $\mathrm{Rx}=+0,06$

2) Protéine $\mathrm{Rx}=+0,20$

3) Protéine $\mathrm{Rx}=+1,21$

4) Protéine $\mathrm{Rx}=-0,60$ (Para-caséine $x$ ).

\section{Fromage Roquefort}

1) Protéine $\mathrm{Rx}=+0,51$

2) Protéine $R x=-0,30$ (Para-caséine $x$ ).

Les enseignements tirés, précédemment de l'examen des électrophorèses des protéines des fromages de vache et de brebis, nous permettent de considérer l'électrophorèse sur gel comme un moyen d'identifier ces produits.

Pour vérifier que cette conclusion est de valeur, nous avons examiné un grand nombre d'échantillons de fromages bleus de vache d'origine et de caractéristiques variées: Bleu des Causses, Bleu de Bresse, Bleus Pasteurisés, Bleu Danois, Stilton, etc. Leur schéma 


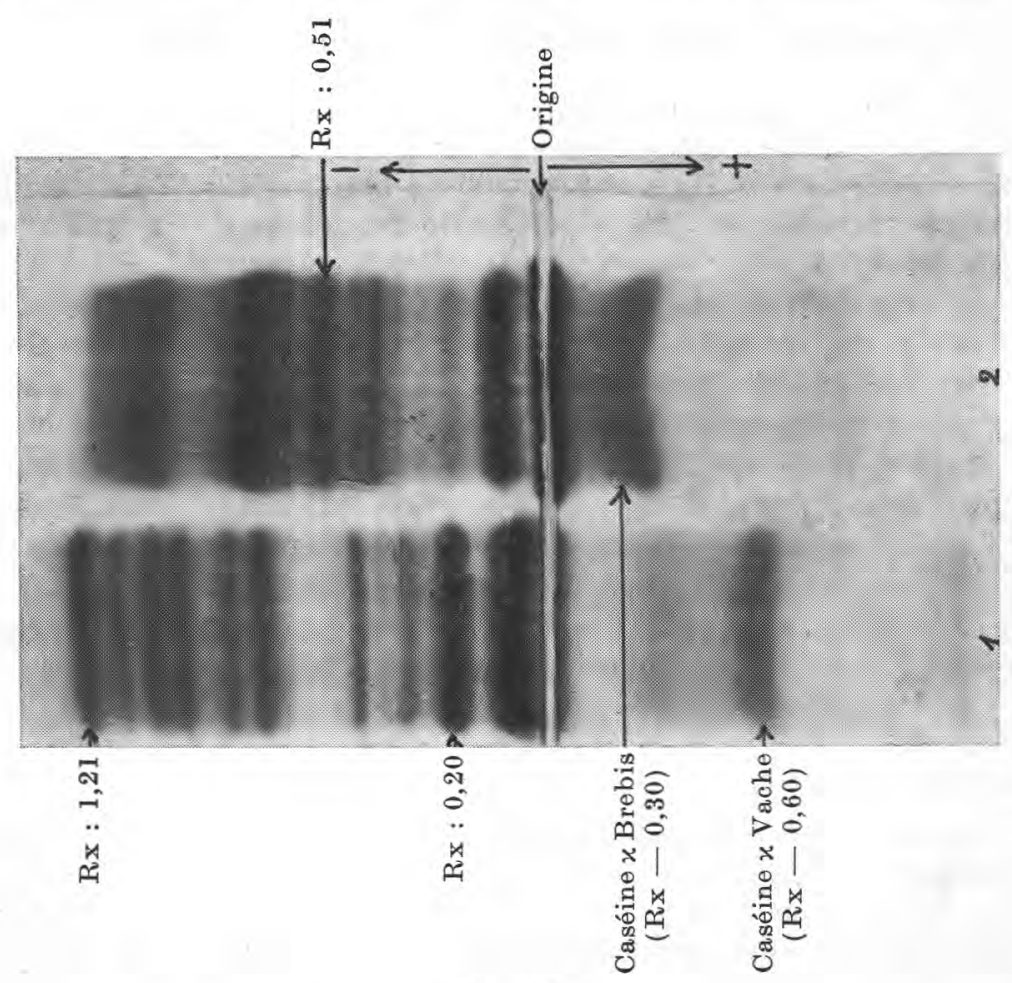

Fig, 7

1) Fromage Bleu de vache

2) Fromage Roquefort

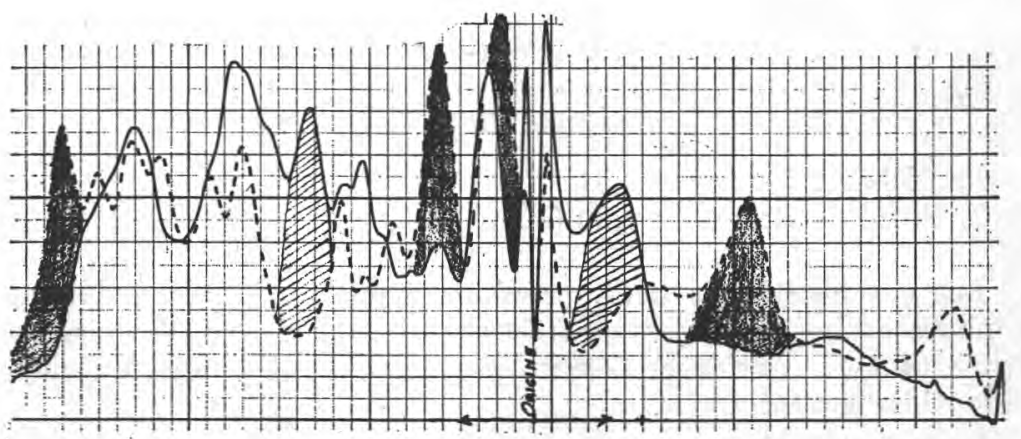

Fig. 8. - Diagrammes d'électrophorèse et substances caractéristiques.

- : Roquefort : en hachuré

- - : Bleu de vache : en grisé 
électrophorétique avait, dans tous les cas, une configuration identique à celle des électrophorèses et électrophorégrammes de Bleu de vache des figures 7 et 8 .

Nous avons fait les mêmes observations sur le Roquefort et les Bleus de brebis fabriqués avec des laits d'origines différentes: Brebis de race Larzac, Lacaune, Sarde, ou Afrique du Nord. La preuve est donc faite que les fromages de vache et de brebis ont bien des caractéristiques qui permettent de les différencier de façon certaine. La différence de nature des caséines du lait se perpétue à un autre niveau dans le fromage.

\section{II. - Fromages Roquefort et Bleu de vache, aux divers stades d'évolution.}

Le développement précédent fait état d'une étude de produits affinés commercialisables. Il convenait en outre d'examiner le fromage aux divers stades de son évolution.

Les figures 9 et 10 regroupent les électrophorèses et électrophorégrammes de l'évolution protéolytique comparée des caséines de Bleu de vache et de Roquefort : fromage frais, fromage en cours d'affinage, fromage affiné.

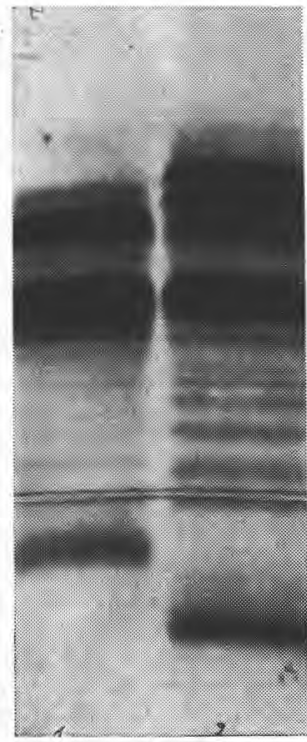

A

Fromage frais

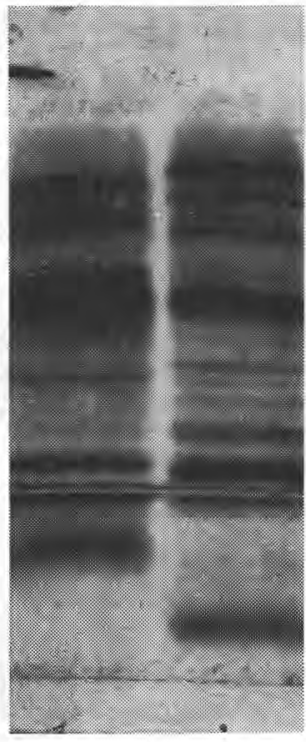

B

fromage demi-affiné

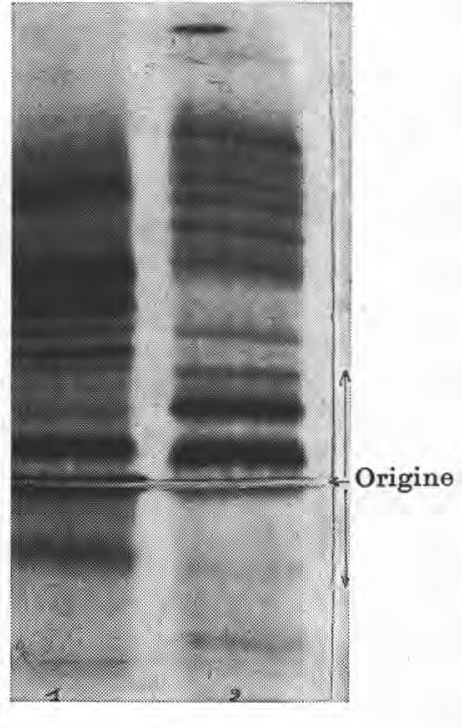

C

fromage affiné

Fig. 9.

(Pour chaque groupe d'électrophorèses et de gauche à droite : fromage de brebis, fromage de vache) 
Nous retiendrons de cet examen :

1) Que les caséines non dégradées conservent leur position d'origine. Notons spécialement la présence des caséines $x$ débarrassées de leur caséino-macropeptide du fait de l'action de la présure.

Para-caséine $x$ vache $\mathrm{Rx}:-0,60$

Para-caséine $x$ brebis $R x:-0,30$

2) Que les positions intermédiaires du processus de la dégradation des protéines s'ordonnent en fonction de la nature des caséines d'origine, et ceci par conséquent de façon spécifique.

3) Que les substances dérivées des caséines apparaissent graduellement pour atteindre un optimum au niveau du fromage commercialisable.

En résumé, à tous les stades de la vie du fromage, la présence de substances protéiniques spécifiques permet d'établir de façon formelle l'identité d'un fromage considéré.

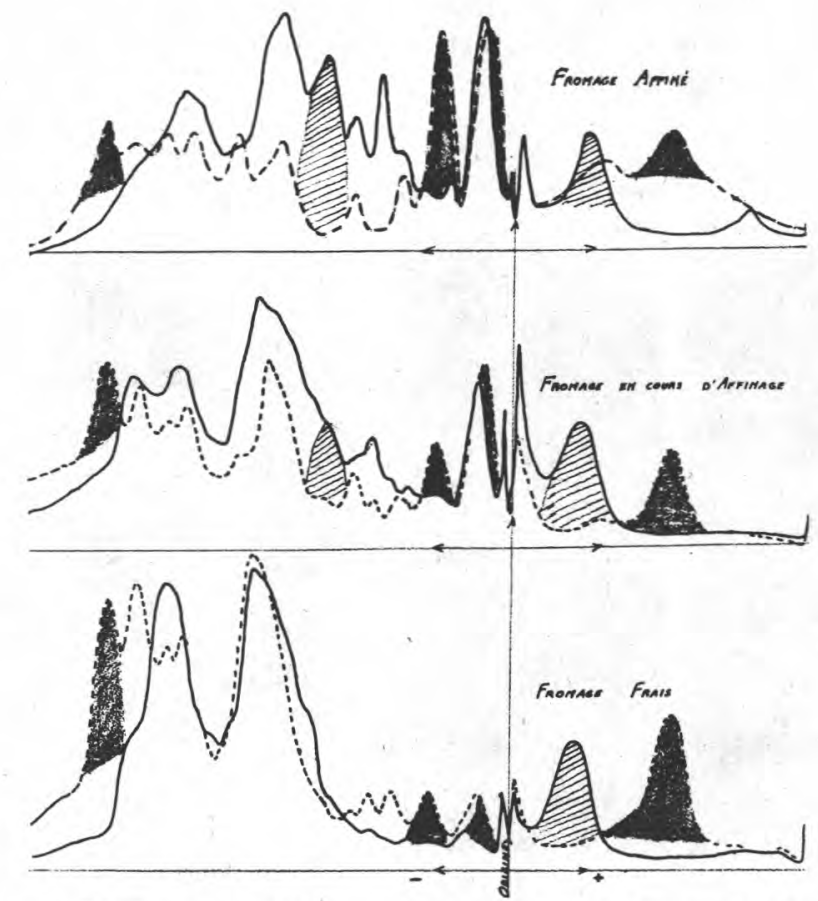

Fig. 10. - Diagrammes d'électrophorèses sur fromage à divers stades d'évolution.

fromage de brebis fromage de vache 
Nature des protéines précipitables.

Cette étude complémentaire avait pour but de nous renseigner sur la nature de ces substances par rapport aux caséines dont elles sont issues. Nous avons utilisé, pour cela, la propriété remarquable des caséines $\alpha_{\mathrm{s}}$ de devenir insolubles à $0^{\circ} \mathrm{C}$ en présence de calcium, alors que les caséines $\beta$ ne précipitent, dans les mêmes conditions, qu'au-dessus de $20^{\circ} \mathrm{C}$.

Nous avons ainsi séparé du fromage de Roquefort et du Bleu de vache affinés, deux catégories de caséines : celles qui avaient un caractère $\alpha_{\mathrm{s}}$ caséine, et celles qui avaient un caractère $\beta$ caséine.

Pour le fromage de vache, nous avons établi que les protéines qui migrent au-delà du $R x 0,70$ (caséines $\alpha_{s}$ ), avaient la réactivité au calcium des caséines $\alpha_{s}$. On peut donc en déduire qu'elles en dérivent. Quant aux dérivés des caséines $\beta$, ils ont des Rx inférieurs à 0,70 .

Pour le fromage de brebis, les caséines $\alpha_{\mathrm{s}}$ donnent comme dérivés toutes les substances de $\mathrm{Rx}$ supérieur à 0,70 et la substance de $\mathrm{Rx}$ de 0,51 . Les substances dérivées à réactivité $\alpha_{\mathrm{S}}$ sont celles qui ont un $\mathrm{Rx}$ inférieur à 0,70 , à l'exception. cependant, de la protéine de $R \times 0,51$.

Comme corollaire à cet ensemble d'observations, il est logique de penser que les dérivés de l'attaque primaire des caséines sont des protéines encore très voisines de ces dernières. Elles ont le caractère de précipitabilité à $p H$ 4,6 et de solubilité à l'urée et réagissent au calcium de façon identique.

Autres fromages.

Nous avons examiné sommairement quelques échantillons de fromages autres que les bleus : Camembert Normand, Carré de l'Est, Reblochon, Port Salut, à base de lait de vache; Manchego, Sarde, Oriental, Laruns, à base de lait de brebis.

Un premier examen nous amène à observer des différences assez nettes entre catégories de fromages de même origine laitière. En fait, les différences observées sont essentiellement dues à des différences dans le niveau de protéolyse de chaque fromage considéré. Si bien que les substances spécifiques à chaque catégorie de laits (caséines natives, substances dérivées) permettent de situer la nature du lait utilisé pour la fabrication du fromage considéré.

Les critères d'identification d'origine vache ou brebis sont donc des éléments fixes. Dans le cours de tels examens il convient seulement de tenir compte des états de protéolyse du fromage, états qui peuvent varier considérablement d'un produit à un autre.

\section{Préparation de fromages. Dressings.}

Il nous a paru intéressant d'étudier les protéines de préparations à base de fromage : fromages fondus et "dressings ». 
L'examen électrophorétique des fromages fondus est pratiqué de la même façon que pour les fromages. Bien que ces produits soient soumis au traitement technologique brutal de la chaleur et des sels de fonte (polyphosphates), les protéines recueillies pour l'électrophorèse ne semblent par avoir subi de modifications.

Les "dressings" sont des sauces-condiments consommées en grande quantité dans les pays anglo-saxons, aux Etats-Unis notamment; le fromage en est un élément de préparation. Leurs formules sont très variées. A titre indicatif, voici l'une d'elles : huile végétale, vinaigre, roquefort, jaune d'œuf, tomate, sucre, oignons, sel, moutarde, jus de citron, câpres, épices, glutamate de sodium. Cette formule comprend du Roquefort. D'autres utilisent des bleus danois, du camembert, etc.

Le problème de l'identification du fromage entrant dans la composition de ces produits paraissait à priori difficile. Il convenait d'abord d'isoler les protéines d'origine fromagère. Mais nous ne pouvions exclure la présence génante de protéines d'autres origines. En fait, les extraits de caséines obtenus sur ces produits, d'après le protocole mentionné plus haút, nous ont fourni des migrations électrophorétiques typiques des fromages. L'examen des spectres obtenus nous a renseigné sur la nature du fromage : Bleu de vache, Roquefort ou mélange. Nous avons cependant remarqué que, dans les produits vieux et dénaturés, toutes les caséines finissaient par disparaître au profit de substances à poids moléculaire plus bas. A partir de cet instant, aucune identification n'est possible par ce procédé.

Nous avons aussi examiné quelques échantillons de fromage de chèvre. Le schéma électrophorétique des caséines de ce fromage présente des caractères de spécificité. Nous n'avons pas approfondi ces examens, mais il semble bien que la méthode électrophorétique puisse jouer également son rôle pour leur identification.

Nous avons noté, par exemple, que la caséine $x$, de Rx-0,06 était spécifique du fromage d'origine chèvre et que cette protéine se maintenait dans ce fromage jusqu'à un stade de dégradation avancée.

(à suivre) 